\title{
Comparison and Analysis of Electronic Voltage Transformer Based on Voltage Detection
}

\author{
Yanxia Wang ${ }^{*}$ and Xingcang Zhai \\ Tuocheng Power Supply Co., Ltd., Shangqiu, Henan 476200, China
}

\begin{abstract}
The electronic voltage Transformer (EVT) constitutes key equipment in the intelligent substation; during the half-a-century development, numerous categories have emerged. By analysis and comparison of the working mechanisms and features of various electronic voltage transformers, we have concluded the strengths and defects in various mechanisms and impact factors on the features of voltage transformer and raised specific measures to reduce the impact and problems found in the design. The findings have revealed that ECVT is the mainstream product in the electronic voltage transformer market but interim measuring accuracy requires for improvement; ERVT is limited by the resistance power and insulating property, and not applicable to the higher voltage level; OVT assures excellent measuring quality in the sensing mechanism. However, the application progress will be slowed down due to several factors: the complex production process, measuring precision easily affected by light power fluctuation and temperature change and transmission by optical fibers with a little effect in simplifying insulation and trimming cost.
\end{abstract}

\author{
KEYWORDS \\ Voltage transformer \\ ECVT \\ ERVT \\ Voltage detection \\ OVT
}

\section{Introduction}

The electronic voltage transformer includes two categories in the detection mechanism: detection voltage type and detection current type. The detection voltage type EVT includes the electronic capacitance voltage transformer (ECVT), electronic resistance voltage transformer (ERVT) and optical voltage transformer (OVT): They refer to Pockels electro-optic effect type OVT and inverse piezoelectric effect type OVT. This paper makes comparison and analysis on the mechanisms and features of said electronic voltage transformers, concludes the strengths and defects of various mechanisms and impact factors on voltage transformer features, and raises notices in the design and measures adopted to reduce impact.

\section{General structure of electronic voltage transformer}

According to the standard definition of IEC, the general

Copyright (๑) 2015 Yanxia Wang and Xingcang Zhai

doi: 10.18686/esta.v2i1.10

Received: October 4, 2015; Accepted: October 30, 2015; Published online: December 4

This is an open-access article distributed under the terms of the Creative Commons Attribution Unported License (http://creativecommons.org/ licenses/by-nc/4.0/), which permits unrestricted use, distribution, and reproduction in any medium, provided the original work is properly cited. ${ }^{*}$ Corresponding author: Tuocheng Power Supply Co., Ltd., Shangqiu, Henan 476200, China. E-mail: wangyanxia009@sina.com structure of electronic voltage transformer is composed of primary part, secondary part and transmission system as shown in Figure 1, P1, P2 refer to primary input ends; according to different detection mechanisms, they can be voltage input or current input [1]. The transmission system is collocated with cable or optical cable; in the digital substation, optical fiber is an ideal choice. The secondary output of analog volume is S1, S2 in order to connect with the traditional metering or protective unit.

According to the primary sensing mechanisms and different combinations of transmission system, EVT general structure's primary component can be neglected in some areas while various procedures of secondary part are not affected. Take optical voltage transformer as an example, since the optical fiber transmission can directly transmit optical measuring signal, primary converter is not needed,

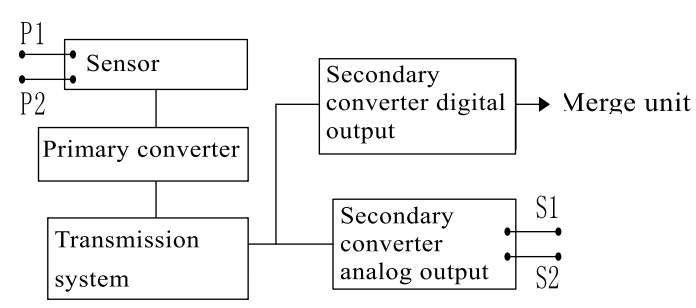

Figure 1. EVT general structure. 
while primary power supply is not needed either. With constant deepening of secondary equipment digitalization, the analog interface of secondary converter is gradually cancelled.

\section{Detection voltage type electronic voltage trans- former \\ 3.1. ECVT}

The ECVT adopts capacitor as the sensor; in spite of slight differences of sensors in the structure (designed by different manufacturers) [2-7], ECVT mechanism and structure are described in Figure 2; the figure neglects the power supply of high/low-voltage side electronic parts.

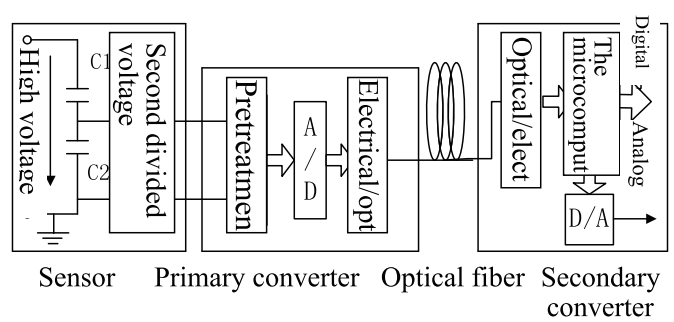

Figure 2. ECVT mechanism and structure.

In the said sensor in the figure, the secondary divided voltage procedure adopts resistance or capacitance. If the power supply is connected at the capacitance C2 side of low-voltage arm in a parallel manner and resistance $\mathrm{R}$ is $1 /$ $\mathrm{R} \omega(\mathrm{C} 1+\mathrm{C} 2)(\omega$-angle frequency of measured voltage), the sensor outputs the differential of measured signal. In the micro-computer treatment, the digital credit is reduced and measured.

ECVT integrates the strengths of capacitance voltage transformer (CVT) and OVT. It is deemed as the mainstream product of EVT. However, it has the following defects:

1) The measuring accuracy is affected by the sundry capacitance and capacitance temperature factor; in the design, capacitance with low temperature factor is selected and adopted, and shield hood is installed in the highvoltage part of transformer to remove or reduce negative impacts of sundry capacitance.

2) ECVT suffers from interim measuring errors: It mainly includes catching of power load and short circuit at the outlet of high-voltage side. Take catching of power load as an example, if the line is switched off, the power load of line equivalent capacitance $\mathrm{C}$ may be caught by ECVT capacitance voltage divider as shown in Figure 3. The catching volume of power load depends on the instantaneous voltage in the switch-off. C2 is connected with equivalent parallel resistance $\mathrm{R}$ of connected equipment to discharge power; power load $\mathrm{Q}$ stored in $\mathrm{C} 1$ is difficult to discharge; if the line is connected for the second time, the line immediately discharges power through low DC impedance of power grid. As a result, power load of $\mathrm{C} 1$ is transferred to $\mathrm{C} 2$ and $\mathrm{C} 2$ is charged to the output value of second- ary voltage. It is attenuated according to the time constant RC2: larger R leads to slower attenuation and longer error time.

3) ECVT may suffer from high EVT secondary voltage [8] in the switch-on of isolating switch; the reason lies in too large equivalent parallel resistances of low-voltage arm, accumulation of residual capacitance load and difficulty in discharge. Therefore, special attention is paid to the equivalent parallel resistance of low-voltage arm in ECVT design.

\subsection{ERVT}

The ERVT differs from the ECVT mainly in the sensor; the precise resistance voltage divider is collocated as the sensing component [9]. The sensing component is featured as mature technology and high measuring accuracy; however, it is limited by the resistance power and insulating property and mainly applicable to medium/low-voltage distribution fields such as $10 \mathrm{kV}$ and $35 \mathrm{kV}$. Figure 4 demonstrates the mechanism and structure of ERVT sensor.

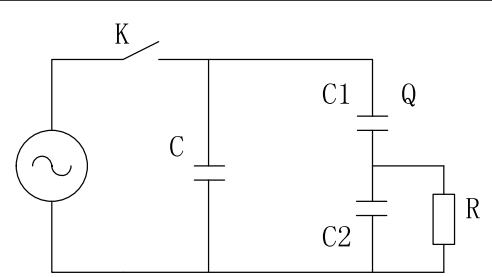

Figure 3. ECVT catching of power load.

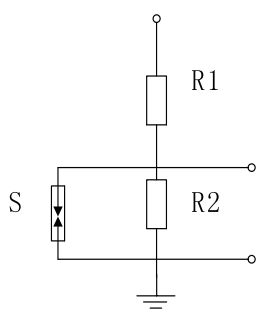

Figure 4. Mechanism and structure of ERVT sensor.

The resistance voltage divider includes the high-voltage arm resistance $\mathrm{R} 1$, low-voltage arm resistance $\mathrm{R} 2$ and over-voltage protective gas discharge pipe $\mathrm{S}$. The measuring quality is mainly affected by the resistance features and sundry capacitance. Highly stably thick film resistance is collocated for $10 \mathrm{kV}$ and $25 \mathrm{kV}$ voltages as the high/lowvoltage arm resistance of voltage divider. In order to block the negative impacts of sundry capacitance, the shield hood is installed to improve the distribution of electric field of voltage divider (same as ECVT).

\subsection{Optical voltage transformer}

In the respect of mechanism, the optical voltage transformer includes OVT based on Pockels electro-optical effect and OVT based on inverter effect. However, both of them make use of the optical crystal and reflect the changed physical volume of electric field strength and thus calculate 
the electric field strength and voltage. Take Pockels electrooptical effect as an example, if the light passes through the electro-optical crystal under the external electric field, double reflection is produced; the phase difference of double reflected light waves is in proportion to the strength of electric field; if the electric field has a fixed distance from the crystal, voltage in the crystal is in proportion. The inverter effect refers to the following condition: If the voltage crystal is affected by the external electric field, it will have certain changes and transfer into the modulation of light signal and inspect the light signal; therefore, it can realize the optical sensing of electric field (or voltage). Figure 5 and Figure 6 demonstrate the mechanism and structure of two OVT sensors.

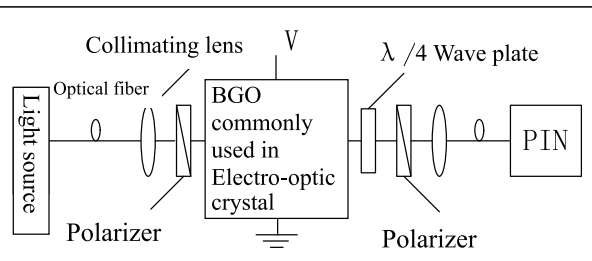

Figure 5. OVT working mechanism based on Pockels effect.

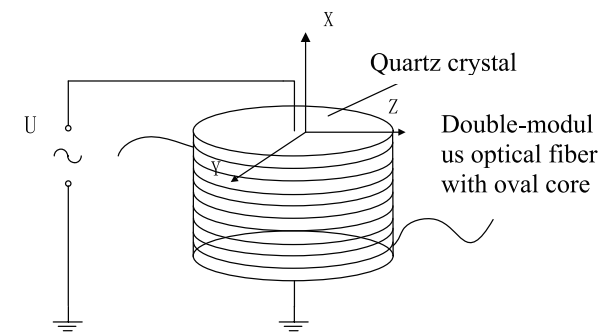

Figure 6. OVT working mechanism based on inverter effect.

The said two figures directly reflect the working mechanism of two OVT. BGO refers to an ideal voltage sensitive material with Pockels electro-optical effect, without natural double reflection, without light rotation and without heat release effect. Therefore, BGO is adopted as the electrooptical crystal. The quartz crystal refers to a voltage crystal; if $\mathrm{AC}$ voltage is imposed along $\mathrm{X}$ axis of cylinder quartz crystal, AC voltage strain is produced in $\mathrm{Y}$ axis so that circumference of cylinder crystal has a certain change. The voltage strain is inspected by double-modulus optical fiber with oval core on the crystal surface; it will reflect the light phase difference of two space models $\left(\mathrm{LP}_{01}\right.$ and $\left.\mathrm{LP}_{11}\right)$ in the transmission.

Compared with voltage divided type EVT, the optical voltage transformer enjoys the greatest OVT practice and industrialization are blocked by the complex production process, impact of fluctuation of light power and change in temperature on measuring precision and negative impact on long-term operation reliability and stability.

\section{Conclusion}

The paper discusses about the electronic voltage transformer in various mechanisms and draws the following conclusions:

(1) Currently, ECVT is the mainstream product of electronic voltage transformer; however, the interim measuring accuracy needs improvement.

(2) ERVT is limited by the resistance power and insulating property and not applicable to the higher voltage level.

(3) OVT assures excellent measuring quality in the sensing mechanism; however, the application progress will be slowed down due to several factors: the complex production process, measuring precision easily affected by light power fluctuation and temperature change and transmission by optical fibers with a little effect in simplifying insulation and trimming cost.

\section{Conflicts of interest}

These authors have no conflicts of interest to declare.

\section{Author's contributions}

These authors contributed equally to this work.

\section{References}

1. Guo, Z. Z. (2008). Introduction and Evaluation of Electronic transformer. Protection and Control of Power System, 36(15), 1-5.

2. Shi, D.G., Liu, Y., \& Zhang, L. P. (2003). Introduction to High-voltage transformer. Transformer, 40(6), 11-14.

3. Wu, T., Zhou, Y.Q., \& Cao, Z. H. (2009). New High/medium-voltage Electronic Voltage transformer. Automatic Power Equipment, 29(12), 109-112.

4. Wang, H. X., Zhang, G. Q., \& Cai, X. G. (2009). Research and Design of Capacitance Voltage Divided Type Voltage transformer. Automatic Power Equipment, 29(10), 83-87.

5. Luo, S. N., \& Nan, Z. L. (2004). Research on Electronic Voltage transformer Based on Capacitance Voltage Differential. High-voltage Technology,30(10), 7-8.

6. Duan, X. Y., Liao, M. F., \& Zou, J. Y. (2003). Research on Electronic Voltage transformer Based on Capacitance Voltage Divider. High-voltage Technology, 29(1), 50-51.

7. Wang, J. Y., Guo, Z. Z., \& Zhang, G. Q. (2012). Simulation and Research of Interim Features of Electronic Voltage transformer. Automatic Power Equipment.32(3), 62-65.

8. Wang, X. B., Jia, H., \& Qian, C. N. (2012). Analysis and Settlement of High Operation Voltage of 220 kV GIS Electronic Voltage transformer. Jilin Power, 40(6):40-42.

9. Fang, C. E., Li, W., Wang, J. Y. (2007). 10kV Electronic Voltage transformer Based on Resistance Divided Voltage. Technical Journal of Electrician, 22(5)58-63. 